\title{
New Robust Stability and Stabilization Conditions for Linear Repetitive Processes
}

\author{
Wojciech Paszke, Olivier Bachelier
}

\begin{abstract}
This paper focuses on the problem of robust stabilization for differential or discrete linear repetitive processes. The provided conditions allow us to involve parameter dependent Lyapunov functions. An additional flexibility in finding a solution is obtained by introducing slack matrix variables. A simulation example is given to illustrate the theoretical developments.
\end{abstract}

\section{INTRODUCTION}

During the last decades, a considerable amount of attention has been paid to robust stability and stabilization of linear systems. Numerous results have been presented, many of them being formulated in terms of linear matrix inequalities (LMIs) (see [12], [7] and the references therein for example). Unfortunately, the main focus is on classical, i.e. 1D, systems and there is only a little number of results in the area of $2 \mathrm{D}$ linear systems [8] and linear repetitive processes (LRPs) [20]. According to this fact, the paper provides such results. Particulary, it is shown that robust analysis and synthesis of differential and discrete repetitive processes become possible through efficient numerical techniques based on LMIs [6].

In this paper we show that there exist a way to introduce an extra matrix variable which allows us to separate the Lyapunov matrices from the process matrices. This property provides a way to derive LMI conditions based on parameter dependent Lyapunov functions [10], [23], [18], [15] that is, is the present work, inspired from what is achieved in 1D systems in [13], [14], [16].

Therefore, following the idea introduced in the abovementioned references, not only the nominal state feedback of design of an LRP is achieved by computing a feedback matrix which is independent on the Lyapynov matrices, but moreover, it is possible to compute this control law while ensuring the robust stability of an uncertain LRP. The uncertainty which is considered in this the paper can be referred to as the polytopic norm-bounded uncertainty. The robustness is guaranteed by LMI-based conditions which are obtained with about the same fashion as in [4]. However, there are several special issues with LRP that deserves careful attention, hence this contribution.

This work is partially carried out as part of CONDOR PROJECT, a project under supervision of the Embedded Systems Institute (ESI) and with FEI company as the industrial partner. This project is partially supported by the Dutch ministry of Economic Affairs under BSIK program.

W. Paszke is with Control Systems Technology Group, Eindhoven University of Technology, P.O. Box 513, 5600 MB Eindhoven, The Netherlands. W.Paszke@tue.nI

Olivier Bachelier is with the University of Poitiers, LAII-ESIP, 40 Avenue du Recteur Pineau, 86022 Poitiers Cedex, France Email: Olivier.Bachelierauniv-poitiers.fr
Throughout this paper, the null matrix and the identity matrix with appropriate dimensions are denoted by 0 and $I$, respectively. Moreover, matrix inequalities are considered in sense of Löwner, i.e. the notation $X \succeq Y$ (respectively $X \succ$ $Y$ ) means that the matrix $X-Y$ is positive semi-definite (respectively, positive definite). In large matrix expressions, the symbol $(\star)$ replaces terms that are induced by symmetry. Expression $M^{H}$ stand for the symmetric matrix $M+M^{T}$. $\|M\|_{2}$ is the matrix 2-norm induced by the Euclidean vector norm. At last, $\operatorname{Ker}(M)$ is the matrix whose columns span the right nullspace of the subset spanned by the columns of $M$.

\section{STABILITY CONDITIONS OF LINEAR REPETITIVE PROCESSES}

In this section, it is dealt with tractable LMI conditions for the stability of nominal linear repetitive processes. Both differential repetitive processes and purely discrete repetitive processes are handled.

Consider a differential LRP of the following form over $0 \leq$ $p \leq \alpha, k \geq 0$,

$$
\begin{aligned}
& \dot{x}_{k+1}(t)=A x_{k+1}(t)+B_{0} y_{k}(t)+B u_{k+1}(t), \\
& y_{k+1}(t)=C x_{k+1}(t)+D_{0} y_{k}(t)+D u_{k+1}(t) .
\end{aligned}
$$

Several equivalent sets of conditions for stability along the pass of the above model are known but here the considered condition is expressed in terms of 2D transfer matrix description of the process dynamics, and hence the 2D characteristic polynomial, which is the basic starting point. Since the state on pass 0 plays no role, it is convenient to re-label the state vector as $x_{k+1}(t) \mapsto x_{k}(t)$ (keeping of course the same interpretation). Also define the pass-to-pass shift operator as $z_{2}$ applied e.g. to $y_{k}(t)$ as follows

$$
y_{k}(t):=z_{2} y_{k+1}(t)
$$

and for the along the pass dynamics, we use the Laplace transform variable $s$, where it is routine to argue that finite pass length $\alpha$ does not cause a problem provided the variables considered are suitably extended from $[0, \alpha]$ to $[0, \infty]$ (here we assume that this has been done).

Let $Y\left(s, z_{2}\right)$ and $U\left(s, z_{2}\right)$ denote the transforms to the sequences $\left\{y_{k}\right\}_{k}$ and $\left\{u_{k}\right\}_{k}$ respectively. Then the process dynamics can be written as

$$
Y\left(s, z_{2}\right)=G_{y u}\left(s, z_{2}\right) U\left(s, z_{2}\right)
$$


where the $2 \mathrm{D}$ transfer function matrix $G_{y u}\left(s, z_{2}\right)$ is given by

$$
G_{y u}\left(s, z_{2}\right)=\left[\begin{array}{ll}
0 & I
\end{array}\right]\left(\left[\begin{array}{cc}
s I-A & -B_{0} \\
-z_{2} C & I-z_{2} D_{0}
\end{array}\right]\right)^{-1}\left[\begin{array}{c}
B \\
D
\end{array}\right]
$$

The $2 \mathrm{D}$ characteristic polynomial is given by

$$
\mathcal{C}\left(s, z_{2}\right):=\operatorname{det}\left(\left[\begin{array}{cc}
s I-A & -B_{0} \\
-z_{2} C & I-z_{2} D_{0}
\end{array}\right]\right)
$$

and it has been shown in [21] that stability along the pass holds if, and only if,

$$
\mathcal{C}\left(s, z_{2}\right) \neq 0 \quad \forall\left\{s, z_{2}\right\} \in\left\{\left(s, z_{2}\right): \operatorname{Re}(\mathrm{s}) \geq 0,\left|\mathrm{z}_{2}\right| \leq 1\right\}
$$

It is also possible to use this $2 \mathrm{D}$ transfer matrix description to conclude that stability along the pass requires each frequency component of the initial profile (and hence on each subsequent pass) to be attenuated from pass to pass.

Also consider the following discrete LRP,

$$
\begin{aligned}
x_{k+1}(p+1) & =A x_{k+1}(p)+B_{0} y_{k}(p)+B u_{k+1}(p) \\
y_{k+1}(p) & =C x_{k+1}(p)+D_{0} y_{k}(p)+D u_{k+1}(p),
\end{aligned}
$$

which can be obtained, for instance, by sampling the along the pass dynamics of the model (1). Again it is necessary to specify the boundary conditions, which are given by

$$
\begin{aligned}
x_{k+1}(0) & =d_{k+1}, k \geq 0, \\
y_{0}(p) & =f(p), p=0,1, \ldots, \alpha-1 .
\end{aligned}
$$

It is clear that for this case, the Laplace variable $s$ should be replaced by some shifting operator $z_{1}$ so that the corresponding transfer matrix $\operatorname{Gyu}\left(z_{1}, z_{2}\right)$ leads to the following 2D characteristic polynomial:

$$
\mathcal{C}\left(z_{1}, z_{2}\right)=\operatorname{det}\left(\left[\begin{array}{cc}
I-z_{1} A & -z_{1} B_{0} \\
-z_{2} C & I-z_{2} D_{0}
\end{array}\right]\right)
$$

Based on the above characteristic polynomial it can be investigated that stability along the pass for discrete LRP holds if, and only if

$$
\mathcal{C}\left(z_{1}, z_{2}\right) \neq 0, \forall\left(z_{1}, z_{2}\right):\left|z_{1}\right| \leq 1,\left|z_{2}\right| \leq 1
$$

Before going on with technical developments, it must be noticed that both the above models can be written as an augmented model model with state vector

$$
\xi_{k}(t)=\left[\begin{array}{c}
x_{k+1}(t) \\
y_{k}(t)
\end{array}\right] \quad \text { or } \quad \xi_{k}(p)=\left[\begin{array}{c}
x_{k+1}(p) \\
y_{k}(p)
\end{array}\right]
$$

so that

$$
\delta \xi_{k+1}=\mathcal{A} \xi_{k}+\mathcal{B} u_{k+1}
$$

where $\delta$ stands for the derivation or shifting operator (depending on the differential or purely discrete case), where

$$
\mathcal{A}=\left[\begin{array}{cc}
A & B_{0} \\
C & D_{0}
\end{array}\right] \text { and } \mathcal{B}=\left[\begin{array}{c}
B \\
D
\end{array}\right] .
$$

However, it is very difficult to provide computationally effective tests for stability in this way. One of the ways to derive tractable tests is by applying Lyapunov theory associated with LMI techniques, that became a standard tool for the stability analysis of 1D systems when manipulating state-space models.

This approach uses the following candidate Lyapunov function for models (1) and (3) respectively,

$$
\begin{aligned}
V(k, t) & =V_{1}(k, t)+V_{2}(k, t) \\
& =x_{k+1}^{T}(t) P_{1} x_{k+1}(t)+y_{k}^{T}(t) P_{2} y_{k}(t) \\
V(k, p) & =V_{1}(k, t)+V_{2}(k, t) \\
& =x_{k+1}^{T}(p) P_{1} x_{k+1}(p)+y_{k}^{T}(p) P_{2} y_{k}(p)
\end{aligned}
$$

for some $P_{1} \succ 0$ and $P_{2} \succ 0$. The above function is a combination of two independent functions due to the twodimensional character of the considered class of system. Since

$$
\begin{aligned}
\dot{V}_{1}(k, t) & =\dot{x}_{k+1}^{T}(t) P_{1} x_{k+1}(t)+x_{k+1}^{T} P_{1} \dot{x}_{k+1}(t) \\
\Delta V_{2}(k, t) & =y_{k+1}^{T}(t) P_{2} y_{k+1}(t)-y_{k}^{T}(t) P_{2} y_{k}(t)
\end{aligned}
$$

or

$$
\begin{aligned}
& \Delta V_{1}(k, t)=x_{k+1}^{T}(p) P_{2} x_{k+1}(p)-x_{k}^{T}(p) P_{2} x_{k}(p) \\
& \Delta V_{2}(k, t)=y_{k+1}^{T}(p) P_{2} y_{k+1}(p)-y_{k}^{T}(p) P_{2} y_{k}(p)
\end{aligned}
$$

respectively, then the associated increment for (8) is calculated as follows:

$$
\Delta V(k, t)=\dot{V}_{1}(k, t)+\Delta V_{2}(k, t)
$$

or

$$
\Delta V(k, p)=\Delta V_{1}(k, p)+\Delta V_{2}(k, p)
$$

respectively. Hence, using the Lyapunov approach, the sufficient condition to guarantee the stability along the pass of a differential or discrete LRP is given by the following corollary to Lyapunov's second method.

Lemma 1. [11] Consider the differential LRP (1) or the discrete LRP (3). Then such a process is stable along the pass (in the sense of Lyapunov) if the following condition

$$
\Delta V(k, t)<0
$$

is satisfied.

Remark 1. It has to be emphasized that stability condition (12) is only sufficient. To see this, recall that the state space model (1) can be embedded into the Roesser [19] or Fornasini-Marchesini [9] model which only deal with the local state unlike the global state which preserves all past information as in $1 D$ case. Furthermore, based on Lyapunov methods, the stability result can be established for local case only. Therefore, negativity of the local increment (10) guarantees stability but with some degree of conservatism.

Condition (12) for stability along the pass can be written in terms of LMI feasibility problem as follows.

Lemma 2. [11] Assume that there exist matrices $P_{1} \succ 0$ and $P_{2} \succ 0$ of compatible dimensions such that LMI

$$
\left[\begin{array}{ccc}
-P_{2} & P_{2} C & P_{2} D_{0} \\
C^{T} P_{2} & A^{T} P_{1}+P_{1} A & P_{1} B_{0} \\
D_{0}^{T} P_{2} & B_{0}^{T} P_{1} & -P_{2}
\end{array}\right] \prec 0
$$


or

$$
\left[\begin{array}{cccc}
-P_{1} & 0 & P_{1} A & P_{1} B_{0} \\
0 & -P_{2} & P_{2} C & P_{2} D_{0} \\
A^{T} P_{1} & C^{T} P_{2} & -P_{1} & 0 \\
B_{0}^{T} P_{1} & D_{0}^{T} P_{2} & 0 & -P_{2}
\end{array}\right] \prec 0
$$

holds, then the differential LRP described by (1) (respectively the discrete LRP described by (3)) is stable along the pass.

Equivalently, i.e. without introducing any additional degree of conservatism, the stability along the pass condition is formulated by the following result

Theorem 1. Assume that there exist matrices $Y_{1} \succ 0, Y_{2} \succ 0$ and $G$ of compatible dimensions such that

$$
\Upsilon+\left(\left[\begin{array}{c}
\mathcal{A} \\
-I
\end{array}\right] G \mathcal{I}\right)^{H} \prec 0
$$

where $\mathcal{A}$ is given by (7), where

$$
\Upsilon=\left[\begin{array}{rrrr}
0 & 0 & Y_{1} & 0 \\
0 & -Y_{2} & 0 & 0 \\
Y_{1} & 0 & 0 & 0 \\
0 & 0 & 0 & Y_{2}
\end{array}\right] \text { or } \Upsilon=\left[\begin{array}{rrrr}
-Y_{1} & 0 & 0 & 0 \\
0 & -Y_{2} & 0 & 0 \\
0 & 0 & Y_{1} & 0 \\
0 & 0 & 0 & Y_{2}
\end{array}\right]
$$

and where

$$
\mathcal{I}=\left[\begin{array}{llll}
I & 0 & I & 0 \\
0 & 0 & 0 & I
\end{array}\right] \text { or } \mathcal{I}=\left[\begin{array}{llll}
0 & 0 & I & 0 \\
0 & 0 & 0 & I
\end{array}\right]
$$

then a differential LRP (1) (respectively the discrete LRP (3)) is stable along the pass.

Proof: The idea is mostly inspired from the works achieved for the 1D-case [13], [14], [16]. It actually consists in applying matrix elimination procedure [22].

Assume that (15) holds. Then applying the above-mentioned procedure shows that (15) is equivalent to

$$
\left(\operatorname{Ker}\left(\mathcal{I}^{T}\right)\right)^{T} \Upsilon \operatorname{Ker}(\mathcal{I}) \prec 0
$$

together with

$$
\left[\begin{array}{ll}
I & \mathcal{A}
\end{array}\right] \Upsilon\left[\begin{array}{c}
I \\
\mathcal{A}^{T}
\end{array}\right] \prec 0,
$$

with noting that (18) always holds. For the differential LRP case, inequality (19) also writes

$$
\left[\begin{array}{cc}
A Y_{1}+Y_{1} A^{T}+B_{0} Y_{2} B_{0}^{T} & Y_{1} C^{T}+B_{0} Y_{2} D_{0}^{T} \\
(\star) & -Y_{2}+D_{0} Y_{2} D_{0}^{T}
\end{array}\right] \prec 0,
$$

which, by virtue of Schur's complement formula, and with permutations on the rows and columns, is equivalent to

$$
\left[\begin{array}{ccc}
-Y_{2} & C Y_{1} & D_{0} Y_{2} \\
(\star) & A Y_{1}+Y_{1} A^{T} & B_{0} Y_{2} \\
(\star) & (\star) & -Y_{2}
\end{array}\right] \prec 0 .
$$

It is very easy to see that the above LMI is the dual condition of LMI (13). An analogous reasoning can be followed for the discrete LRP case in order to recover LMI (14).

The above result introduces extra degree of freedom (by means of the matrix $G$ ) which will not only be used for synthesis purpose but which will reveal particularly useful in the robust context to introduce parameter-dependent Lyapunov functions.

\section{STABILIZATION OF NOMINAL LRP}

Assume that the model (1) or (3), i.e. augmented model (6), is subject to the static state feedback control law described by

$$
u_{k+1}=\left[\begin{array}{ll}
K_{1} & K_{2}
\end{array}\right]\left[\begin{array}{c}
x_{k+1} \\
y_{k}
\end{array}\right]=K \xi_{k},
$$

where $K_{1}$ and $K_{2}$ are appropriately dimensioned matrices to be designed. In effect, this control law uses feedback of the current state vector (which is assumed to be available for use) and 'feedforward' of the previous pass profile vector. Note that in repetitive processes, the term 'feedforward' is used to describe the case where state or pass profile information from the previous pass (or passes) is used as (part of) the input to a control law applied on the current pass, i.e. to information which is propagated in the pass-to-pass $(k)$ direction. The induced closed-loop model becomes

$$
\begin{aligned}
& \dot{x}_{k+1}(t)=\left(A+B K_{1}\right) x_{k+1}(t)+\left(B_{0}+B K_{2}\right) y_{k}(t), \\
& y_{k+1}(t)=\left(C+D K_{1}\right) x_{k+1}(t)+\left(D_{0}+D K_{2}\right) y_{k}(t),
\end{aligned}
$$

for the differential LRP or

$$
\begin{aligned}
& x_{k+1}(p+1)=\left(A+B K_{1}\right) x_{k+1}(p)+\left(B_{0}+B K_{2}\right) y_{k}(p), \\
& y_{k+1}(p+1)=\left(C+D K_{1}\right) x_{k+1}(p)+\left(D_{0}+D K_{2}\right) y_{k}(p),
\end{aligned}
$$

for the discrete LRP, which, in any case, can be written

$$
\delta \xi_{k}=\mathcal{A}_{c} \xi_{k},
$$

where the associated closed-loop state matrix is

$$
\mathcal{A}_{c}=\mathcal{A}+\mathcal{B} K
$$

with $\mathcal{A}$ and $\mathcal{B}$ given by (7).

Assuming that the open-loop process (1) or (3) is unstable along the pass, the question is is to know if there exists some control law complying with (22) that makes model (25) become stable along the pass. Following the same idea as in [14], the next theorem is stated.

Theorem 2. Let a repetitive process be described by (1) or (3), i.e. by (6), then there exists a state feedback control control law (22) stabilizing the process along the pass if there exist matrices $Y_{1} \succ 0, Y_{2} \succ 0, G$ and $L$ such that

$$
\Upsilon+\left(\left(\left[\begin{array}{c}
\mathcal{A} \\
-I
\end{array}\right] G+\left[\begin{array}{c}
\mathcal{B} \\
0
\end{array}\right] L\right) \mathcal{I}\right)^{H} \prec 0
$$

with $\mathcal{A}, \mathcal{B}, \Upsilon$ and $\mathcal{I}$ given by (7), (16) and (17). In this event, the gain matrix is given by

$$
K=L G^{-1} \text {. }
$$

Proof: According to (28), just substitute $L$ with $K G$ in (27) to see that condition (15) is recovered but with $\mathcal{A}_{c}$ rather than $\mathcal{A}$ and thus, by virtue of Theorem 1, model (25) is stable along the pass.

It is interesting to note that, unlike previous results where matrices $K_{1}$ and $K_{2}$ are directly deduced from dual Lyapunov matrices $Y_{1}$ and $Y_{2}$, matrix $K$ is here computed directly owing to $L$ and $G$ which might bring additional flexibility, reducing conservatism, especially in the uncertain case. 


\section{ROBUST STABILIZATION OF LRPS}

The interest in introducing matrix $G$ is not only to bring flexibility in the design but also to enable the implicit involvement of parameter-dependent Lyapunov functions when the model is itself parameter-dependent.

Indeed, assume that the model (6) is actually subject to uncertainty such that it can be written

$$
\delta \xi_{k+1}=\mathbb{A} \xi_{k}+\mathbb{B} u_{k+1}
$$

where

$$
\left[\begin{array}{ll}
\mathbb{A} & \mathbb{B}
\end{array}\right]=\left[\begin{array}{ll}
\mathcal{A} & \mathcal{B}
\end{array}\right]+\left[\begin{array}{ll}
\Delta_{A} & \Delta_{B}
\end{array}\right]
$$

In the above matrix, it is assumed that matrices $\mathcal{A}$ and $\mathcal{B}$ are actually dependent on a real parameter vector $\theta$ with a polytopic dependency:

$$
\left[\begin{array}{ll}
\mathcal{A} & \mathcal{B}
\end{array}\right]=\left[\begin{array}{ll}
\mathcal{A}(\theta) & \mathcal{B}(\theta)
\end{array}\right]=\sum_{i=1}^{N} \theta_{i}\left[\begin{array}{ll}
\mathcal{A}_{i} & \mathcal{B}_{i}
\end{array}\right], \quad \theta \in \Theta
$$

where

$$
\Theta=\left\{\theta=\left[\begin{array}{c}
\theta_{1} \\
\vdots \\
\theta_{N}
\end{array}\right]: \theta_{i} \geq 0, \sum_{i=1}^{N} \theta_{1}=1\right\}
$$

It means that the various matrices $\left[\mathcal{A}_{i} \mathcal{B}_{i}\right]$, which are known, are the vertices of a polytope in which the actual matrix $[\mathcal{A} \mathcal{B}]$ lies.

Moreover the additive uncertainties obey, as in [17], to

$$
\left\{\begin{aligned}
\Delta_{A} & =H_{A} \mathcal{F}_{A} E_{A}, & & \left\|\mathcal{F}_{A}\right\|_{2} \leq \rho_{A}, \\
\Delta_{B} & =H_{B} \mathcal{F}_{B} E_{B}, & & \left\|\mathcal{F}_{B}\right\|_{2} \leq \rho_{B} .
\end{aligned}\right.
$$

Matrices $H_{A}, H_{B}, E_{A}$ and $E_{B}$ are introduced to give some desired structure to the additive uncertainty and $\mathcal{F}_{A}$ and $\mathcal{F}_{B}$ are uncertain matrices that belong to some balls of matrices whose respective radii are $\rho_{A}$ and $\rho_{B}$. To make this description even more general, it is assumed that matrices $H_{A}, H_{B}, E_{A}$ and $E_{B}$ are also submitted to a polytopic dependency:

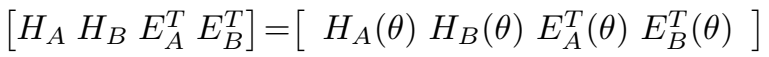

$$
\begin{aligned}
& =\sum_{i=1}^{N} \theta_{i}\left[\begin{array}{llll}
H_{A_{i}} & H_{B_{i}} & E_{A_{i}}^{T} & E_{B_{i}}^{T}
\end{array}\right], \theta \in \Theta .
\end{aligned}
$$

Such an uncertainty is referred to as the polytopic normbounded uncertainty (see [4] or [3]). It very easy to extend such a description to the polytopic LFT-based uncertainty with norm-bonded constraint as in [5]. However, this extension is not presented here to avoid heaviness.

With the control law (35), the uncertain closed-loop model becomes

$$
\delta \xi_{k}=\left(\mathcal{A}_{c}+\Delta_{A}+\Delta_{B} K\right) \xi_{k}=\mathbb{A}_{c}(\theta) \xi_{k},
$$

where $\mathcal{A}_{c}=\mathcal{A}+\mathcal{B} K$ inherits from the polytopic structure of $\mathcal{A}$ and $\mathcal{B}$. It is however false to claim that $\mathbb{A}_{c}$ is polytopic.
Definition 1. The uncertain closed-loop repetitive process described by (35) is robustly stable along the pass if and only if it is stable along a pass for any value of $\theta$ in $\Theta$ and for any matrices $\mathcal{F}_{A}$ and $\mathcal{F}_{B}$ such that $\left\|\mathcal{F}_{A}\right\|_{2} \leq \rho_{A}$, $\left\|\mathcal{F}_{B}\right\|_{2} \leq \rho_{B}$.

From this definition, the problem of state feedback robust stabilization is to find a gain matrix $K$ such that (35) is robustly stable along the pass.

Theorem 3. Let a repetitive process be described by (29), with (30)-(34). It is robustly stabilizable along the pass by a control law complying with (22) if there exist matrices $Y_{1_{i}} \succ 0, Y_{2_{i}} \succ 0, i=1, \ldots, N$, as well as matrices $G$ and $L$ such that

$$
\begin{aligned}
M_{i}= & {\left[\begin{array}{c|c|c}
Q_{i} & \mathcal{I}^{T}\left[\begin{array}{cc}
G^{T} E_{A_{i}}^{T} & 0 \\
0 & L^{T} E_{B_{i}}^{T}
\end{array}\right] & {\left[\begin{array}{cc}
H_{A_{i}} & H_{B_{i}} \\
0 & 0
\end{array}\right]} \\
\hline(\star) & {\left[\begin{array}{cc}
-I & 0 \\
0 & -I
\end{array}\right]} & 0 \\
\hline(\star) & (\star) & {\left[\begin{array}{cc}
-\rho_{A}^{-2} & 0 \\
0 & -\rho_{B}^{-2}
\end{array}\right]}
\end{array}\right] \prec 0, } \\
& \forall i \in\{1, \ldots, N\},
\end{aligned}
$$

where

$$
Q_{i}=\Upsilon_{i}+\left(\left[\begin{array}{c}
\mathcal{A}_{i} G+\mathcal{B}_{i} L \\
-I
\end{array}\right] \mathcal{I}\right)^{H}
$$

with $\Upsilon_{i}$ defined by indexing $Y_{1}$ and $Y_{2}$ in (16). In this event, the gain matrix $K$ is given by (28).

Proof: Consider the following convex combination:

$$
M(\theta)=M=\sum_{i=1}^{N} \theta_{i} M_{i}
$$

which is clearly negative definite. $M$ can be written (omitting the dependence on $\theta$ for conciseness)

$M=\left[\begin{array}{c|c|c|c}Q & \mathcal{I}^{T}\left[\begin{array}{cc}G^{T} E_{A}^{T} & 0 \\ 0 & L^{T} E_{B}^{T}\end{array}\right] & {\left[\begin{array}{cc}H_{A} & H_{B} \\ 0 & 0\end{array}\right]} \\ \hline(\star) & {\left[\begin{array}{cc}-I & 0 \\ 0 & -I\end{array}\right]} & 0 \\ \hline(\star) & (\star) & {\left[\begin{array}{cc}-\rho_{A}^{-2} & 0 \\ 0 & -\rho_{B}^{-2}\end{array}\right]}\end{array}\right] \prec 0$.

Here, it has to be noticed that dual Lyapunov matrices involved in $\Upsilon$ and thus in $Q$ also depend on $\theta$ in a polytopic way, i.e.

$$
\left[\begin{array}{l}
Y_{1} \\
Y_{2}
\end{array}\right]=\left[\begin{array}{c}
Y_{1}(\theta) \\
Y_{2}(\theta)
\end{array}\right]=\sum_{i=1}^{N} \theta_{i}\left[\begin{array}{c}
Y_{1_{i}} \\
Y_{2_{i}}
\end{array}\right], \quad \theta \in \Theta
$$

Define the matrix $S$ as follows:

$$
S=\left[\begin{array}{cc|cc|cc}
I & \mathbb{A}_{c} & H_{A} \mathcal{F}_{A} & H_{B} \mathcal{F}_{B} & 0 & 0 \\
0 & 0 & 0 & 0 & I & 0 \\
\hline 0 & 0 & 0 & 0 & 0 & I
\end{array}\right] .
$$

Left and right multiplying (38) by $S$ and $S^{T}$ respectively and taking (28) into account leads to

$$
\left[\begin{array}{c|cc}
Z & H_{A} & H_{B} \\
\hline H_{A}^{T} & -\rho_{A}^{-2} I & 0 \\
H_{B}^{T} & 0 & -\rho_{B}^{-2} I
\end{array}\right] \prec 0,
$$


with

$Z=\left[\begin{array}{ll}I & \mathbb{A}_{c}\end{array}\right] \Upsilon\left[\begin{array}{c}\mathbb{A}_{c}^{T} \\ I\end{array}\right]-H_{A} \mathcal{F}_{A} \mathcal{F}_{A}^{T} H_{A}^{T}+H_{B} \mathcal{F}_{B} \mathcal{F}_{B}^{T} H_{B}^{T}$,

which, by virtue of Shur's lemma (applied twice), yields

$$
\begin{aligned}
& {\left[\begin{array}{ll}
I & \mathbb{A}_{c}
\end{array}\right] \Upsilon\left[\begin{array}{c}
\mathbb{A}_{c}^{T} \\
I
\end{array}\right] \prec} \\
& H_{A}\left(\mathcal{F}_{A} \mathcal{F}_{A}^{T}-\rho_{A}^{2} I\right) H_{A}^{T}+H_{B}\left(\mathcal{F}_{B} \mathcal{F}_{B}^{T}-\rho_{B}^{2} I\right) H_{B}^{T} .
\end{aligned}
$$

Due to (33), the right hand side member of the above inequality is negative of zero, what proves that

$$
\left[I \mathbb{A}_{c}\right] \Upsilon\left[\begin{array}{c}
\mathbb{A}_{c}^{T} \\
I
\end{array}\right] \prec 0
$$

From this inequality, it suffices to follow the same reasoning as in the proof of Theorem 1 from equation (19), but with $\mathbb{A}_{c}$ substituting $A$, to prove the closed-loop stability.

Since condition (36) is an LMI, whose feasibility can be checked thanks to any LMI software, it is also possible, using the same software, to solve the convex programming problem that consists in minimizing the objective function

$$
J=\alpha_{A} \rho_{A}^{-2}+\alpha_{B} \rho_{B}^{-2}
$$

(for given positive scalars $\alpha_{A}$ and $\alpha_{B}$ ) under LMI constraint (36). However, we rather advise to fix the value of $\rho_{A}$ (or $\rho_{B}$ ) and to minimize $J=\rho_{B}^{-2}$ (resp. $J=\rho_{A}^{-2}$ ).

The dual Lyapunov matrices $Y_{1}$ and $Y_{2}$ are given by (39) which means that they are parameter-dependent. Such a dependence is allowed by the slack variable $G$, as well introduced for conventional models by [13], [14]. The interest is to avoid the use on constant Lyapunov matrices over the polytope (the so-called quadratic stability along the pass) which is expected to be too conservative. Indeed, in the case of differential LRP, condition (36) gives less conservative results than a quadratic stability-based condition but only from a statistical point of view. To be convinced, it suffices to generate numerous random uncertain LRP models to be stabilized and to compute to percentages of success (following the idea of the numerical evaluation proposed in [1]). Then it will appear that, very often, the parameter-dependent approach succeeds when the quadratic approach fails (or that the optimal criterion $J$ is lower when using a parameter-dependent approach). Nevertheless, counterexamples can be found. In the case of purely discrete LRP, it is possible to use arguments borrowed from [13], [14], [2] to rigorously prove that (36) is always less conservative than a quadratic stability-based condition. This reasoning is not reproduced here because of space reason and since the LMI condition for quadratic stability has not been presented. Nevertheless, one can present the main idea by simply considering the polytopic uncertainty (i.e. omitting the norm-bounded part). The uncertain LRP with (31) and (32) is quadratically stable if and only if

$$
\left[\begin{array}{cc}
-\mathcal{Y} & \mathcal{A Y} \\
(\star) & -\mathcal{Y}
\end{array}\right] \prec 0
$$

with

$$
\mathcal{Y}=\left[\begin{array}{cc}
Y_{1} & 0 \\
0 & Y_{2}
\end{array}\right] \succ 0 \text {. }
$$

(This is the dual condition of (14) with a constant $\mathcal{Y}$ and with a polytopic uncertain matrix $\mathcal{A}$; therefore, it could be simply considered as a definition of quadratic stability). By Schur's complement formula, the above inequality amounts to

$$
-\mathcal{Y}+\mathcal{A} \mathcal{Y} \mathcal{A}^{T} \prec 0 .
$$

Since the uncertainty set is compact, one can find a scalar $\epsilon>0$ small enough such that

$$
-\mathcal{Y}+\mathcal{A} \mathcal{Y} \mathcal{A}^{T}+\epsilon \mathcal{A} \mathcal{Y} \mathcal{A}^{T} \prec 0
$$

which, by Schur's complement formula again, is equivalent to

$$
\begin{gathered}
{\left[\begin{array}{rr}
-\mathcal{Y} & \mathcal{A}(1+\epsilon) \mathcal{Y} \\
(\star) & -(1+\epsilon) \mathcal{Y}
\end{array}\right] \prec 0} \\
\Leftrightarrow\left[\begin{array}{cc}
-\mathcal{Y} & \mathcal{A}(1+\epsilon) \mathcal{Y} \\
(\star) & (1-2(1+\epsilon)+\epsilon) \mathcal{Y}
\end{array}\right] \prec 0 \\
\Leftrightarrow\left[\begin{array}{cc}
-\mathcal{Y} & 0 \\
0 & \mathcal{Y}
\end{array}\right]+\left(\left[\begin{array}{c}
\mathcal{A} \\
-I
\end{array}\right](1+\epsilon) \mathcal{Y} \mathcal{I}\right)^{H}+\left[\begin{array}{cc}
0 & 0 \\
0 & \epsilon \mathcal{Y}
\end{array}\right] \prec 0 .
\end{gathered}
$$

Since the last term in the left hand side member is positive semi-definite, it can be deduced that

$$
\left[\begin{array}{cc}
-\mathcal{Y} & 0 \\
0 & \mathcal{Y}
\end{array}\right]+\left(\left[\begin{array}{c}
\mathcal{A} \\
-I
\end{array}\right] G \mathcal{I}\right)^{H} \prec 0
$$

with $G=(1+\epsilon) \mathcal{Y}$ and $\mathcal{I}$ corresponding to the second definition in (17). The reasoning is analogous when normbounded uncertainties are involved.

In the restrictive case where $H_{A}=H_{B}$ or $E_{A}=E_{B}$, it might be possible to consider only one matrix $\mathcal{F}=\mathcal{F}_{A}=$ $\mathcal{F}_{B}$ such that $\|\mathcal{F}\|_{2} \leq \rho$. This might slightly reduce the conservatism of (36).

\section{Numerical EXAmple}

To illustrate our result, consider the problem of computing the static controller (i.e. the matrix $K$ defined in (22)) for the differential process with uncertainty structure (29)-(35) where

$$
\begin{aligned}
& {\left[\mathcal{A}_{1} \mid \mathcal{B}_{1}\right]=\left[\begin{array}{lll|l}
0.1086 & 0.1538 & 0.0908 & 1.7362 \\
0.0601 & 0.0694 & 0.0823 & 0.3614 \\
0.0598 & 0.0084 & 0.1438 & 1.9599
\end{array}\right],} \\
& {\left[\mathcal{A}_{2} \mid \mathcal{B}_{2}\right]=\left[\begin{array}{lll|l}
1.0860 & 1.5380 & 0.9084 & 1.9292 \\
0.6008 & 0.6944 & 0.8235 & 0.4016 \\
0.5982 & 0.0837 & 1.4385 & 2.1777
\end{array}\right],} \\
& {\left[\mathcal{A}_{3} \mid \mathcal{B}_{3}\right]=\left[\begin{array}{lll|l}
1.3032 & 1.8457 & 1.0901 & 2.3150 \\
0.7210 & 0.8333 & 0.9881 & 0.4819 \\
0.7178 & 0.1004 & 1.7261 & 2.6132
\end{array}\right]}
\end{aligned}
$$

and the norm-boud uncertainty is modelled with

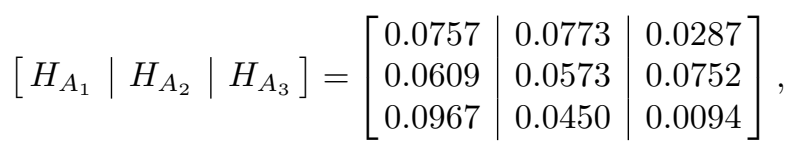




$$
\begin{aligned}
{\left[H_{B_{1}}\left|H_{B_{2}}\right| H_{B_{3}}\right]=\left[\begin{array}{l|l|l}
0.0107 & 0.0581 & 0.0360 \\
0.0735 & 0.0299 & 0.0718 \\
0.0355 & 0.0714 & 0.0995
\end{array}\right], } \\
E_{A_{1}}=\left[\begin{array}{lll}
0.0041 & 0.0329 & 0.0709
\end{array}\right], E_{B_{1}}=0.0161 \\
E_{A_{2}}=\left[\begin{array}{lll}
0.0860 & 0.0501 & 0.0995
\end{array}\right], E_{B_{2}}=0.0159 \\
E_{A_{3}}=\left[\begin{array}{lll}
0.0429 & 0.0267 & 0.0473
\end{array}\right], E_{B_{3}}=0.0268 .
\end{aligned}
$$

Then performing the design procedure of Theorem (3) in Matlab for $\rho_{A}=0.1$ and $\rho_{B}=0.1$ gives the solution as

$$
\begin{aligned}
G & =\left[\begin{array}{ccc}
-397.2800 & -159.6062 & 10.8490 \\
-560.0837 & -237.1113 & -2.5830 \\
198.1517 & 79.5747 & -4.1740
\end{array}\right], \\
L & =\left[\begin{array}{lll}
-0.1723 & 0.2542 & -0.1162
\end{array}\right]
\end{aligned}
$$

and the corresponding controller matrix is

$$
K=\left[\begin{array}{ll}
-0.1961 & -0.0255 \mid-0.4661
\end{array}\right] \text {. }
$$

\section{CONCLUSIONS}

This paper has developed substantial new results on the relatively open problem of robust control of linear repetitive processes which are a distinct class of 2D linear systems of both systems theoretic and applications interest. Particularly, it has been shown that it is possible to introduce an extra matrix variable which allows us to separate the Lyapunov matrices from the process matrices and then give us a way to derive LMI conditions based on parameter dependent Lyapunov functions. Also it has been shown that this result can be extended to the case of a quite general affecting uncertainty affecting the model, namely the polytopic normbounded uncertainty. Here, this is assumed that this uncertainty is present on both the state dynmaics and the pass profile updating equations of the defining state space model. Finally the theoretical findings have been illustrated by the numerical example.

\section{REFERENCES}

[1] O. Bachelier, J. Bernussou, M. C. Oliveira, and J. C. Geromel, "Parameter dependent Lyapunov control design: Numerical evaluation," in Proc. 38th IEEE Conf. Decision and Control(CDC), vol. 1, Phoenix, USA, 7-10 December 1999, pp. 293-297.

[2] O. Bachelier, D. Peaucelle, D. Arzelier, and J. Bernussou, "A precise robust matrix root-clustering analysis with respect to polytopic uncertainty," in Proc. American Control Conference (ACC), vol. 5, Chicago, USA, 28-30 July 2000, pp. 3331-3335.

[3] J. Bosche, O. Bachelier, and D. Mehdi, "Placement de pôles roboratif par retour statique d'état," APII-JESA: Journal Européen des Systèmes Automatisés, vol. 36, no. 10, pp. 1413-1437, 2002.

[4] — , "An approach for robust matrix root-clustering analysis in a union of regions," IMA Journal of Mathematical Control and Information, vol. 22, no. 3, pp. 227-239, 2005.

[5] J. Bosche, R. Riadi, O. Bachelier, D. Mehdi, and L. Rambault, "Robust pole placement by output feedback using a genetic algorithm," in Proc. Conf. Control Applications (CCA), Toronto, Canada, 2005.

[6] S. Boyd, L. E. Ghaoui, E. Feron, and V. Balakrishnan, Linear Matrix Inequalities in System and Control Theory, ser. SIAM Studies in Applied and Numerical Mathematics. Philadelphia, USA: SIAM, 1994, vol. 15.

[7] M. Chilali, P. Gahinet, and P. Apkarian, "Robust pole placement in LMI regions," IEEE Transactions on Automatic Control, vol. 44, no. 12 , pp. 2257-2270, 1999.

[8] C. Du and L. Xie, $\mathcal{H}_{\infty}$ Control and Filtering of Two-dimensional Systems, ser. Lecture Notes in Control and Information Sciences. Berlin, Germany: Springer-Verlag, 2002, vol. 278.
[9] E. Fornasini and G. Marchesini, "State-space realization theory of twodimensional filters,' IEEE Transactions on Automatic Control, vol. 21, no. 4, pp. 484-492, 1976.

[10] P. Gahinet, P. Apkarian, and M. Chilali, "Affine parameter-dependent Lyapunov functions and real parametric uncertainty," IEEE Transactions on Automatic Control, vol. 41, no. 3, pp. 436-442, 1996.

[11] K. Gałkowski, W. Paszke, E. Rogers, S. Xu, J. Lam, and D. H. Owens, "Stability and control of differential linear repetitive processes using an LMI seting," IEEE Transactions on Circuits and Systems - II: Analog and Digital Signal Processing, vol. 50, no. 9, pp. 662-666, 2003.

[12] G. Garcia, J. Bernussou, J. Daafouz, and D. Arzelier, "Robust quadratic stabilization," in Robust Flight Control: A design challenge, ser. Lecture Notes in Control and Information Sciences. SpringerVerlag, 1997, vol. 224, ch. 5.

[13] J. C. Geromel, M. C. de Oliveira, and L. Hsu, "LMI characterization of structural and robust stability," Linear Algebra and its Applications, vol. 285 , no. 1-3, pp. 69-80, 1998.

[14] M. C. d. Oliveira, J. Bernussou, and J. C. Geromel, "A new discretetime robust stability condition," Systems and Control Letters, vol. 39, no. 4, pp. 261-265, 1999.

[15] D. Peaucelle and D. Arzelier, "Robust performance analysis with LMI-based methods for real parametric uncertainty via parameterdependent Lyapunov functions," IEEE Transactions on Automatic Control, vol. 46, no. 4, pp. 624-630, 2001.

[16] D. Peaucelle, D. Arzelier, O. Bachelier, and J. Bernussou, "A new robust D-stability condition for real convex polytopic uncertainty," System and Control Letters, vol. 40, no. 1, pp. 21-30, 2000.

[17] I. R. Petersen, "A stabilization algorithm for a class of uncertain linear systems," Systems and Control Letters, vol. 8, pp. 351-357, 1987.

[18] D. C. W. Ramos and P. L. D. Peres, "A less conservative LMI condition for robust stability of discrete-time uncertain systems," System and Control Letters, vol. 43, no. 5, pp. 371-378, 2001.

[19] R. P. Roesser, "A discrete state-space model for linear image processing," IEEE Transactions on Automatic Control, vol. 20, no. 1, pp. $1-10,1975$.

[20] E. Rogers, K. Gałkowski, and D. H. Owens, Control Systems Theory and Applications for Linear Repetitive Processes, ser. Lecture Notes in Control and Information Sciences. Berlin, Germany: Springer-Verlag, 2007, vol. 349.

[21] E. Rogers and D. H. Owens, Stability Analysis for Linear Repetitive Processes, ser. Lecture Notes in Control and Information Sciences. Berlin, Germany: Springer-Verlag, 1992, vol. 175.

[22] R. E. Skelton, T. Iwasaki, and K. Grigoriadis, A Unified Algebraic Approach to Linear Control Design, ser. Series in Systems and Control. London, England: Taylor and Francis, 1998.

[23] A. Trofino, "Parameter-dependent Lyapunov functions for a class of uncertain linear systems: an LMI approach," in Proc. 38th IEEE Conf. Decision and Control(CDC), vol. 1, Phoenix, USA, 7-10 December 1999, pp. 2341-2346. 